\title{
Enhance Crawler: A Dual-Stage Crawler for Efficiently Harvesting Deep Web Interfaces
}

\author{
Sujata R. Gutte \\ M.E. CSE Dept \\ M. S. Bidwe Egineering College, Latur
}

\author{
Shubhangi S. Gujar \\ Assistant Professor \\ M. S. Bidwe Engineering College, Latur
}

\begin{abstract}
Internet is became important part of our day to day life. That's why due to heavy usage of internet very large amount of diverse data is spread over it and which provide access to search particular data. Very challenging issue for search engine is 'fetch most relevant data as per users need. So to reduce large amount of time spend on searching most relevant data as per user's need. We proposed the "enhanced crawler "In this proposed approach framework is divided into two stages. in the first stage, for center pages search engine perform site based searching for getting more accurate result of focus crawler (it avoid to visit large no of pages ) And ranking is used for prioritize highly relevant ones for given input topic. IN second stage of framework, In-site searching is done by extracting most relevant links with an adaptive link ranking. We design link tree data structure to achieve wider coverage of deep website.
\end{abstract}

\section{Keywords}

Enhance crawler, deep website, ranking, adaptive learning.

\section{INTRODUCTION}

Now a days, The main or the most general approach for accessing and finding information sources is search queries over general search engines such as Google, yahoo or bing. World Wide Web continuously grows, which stores all useful information. URL from the frontier are recursively visited according to a bunch of policies. If crawler performing archiving of web site copies and stores the information as it goes. The achieves are usually stored in such a way they can be viewed. Read and navigated as they were on the live web , but are preserved as snap shorts. Search engine don't cover all the data available on net i.e. there is no such search engines that cover all the web pages existing on the World Wide Web . Typically they ignore valuable data in text database that are hidden behind the search interfaces so those contents are not directly available for crawling through hyperlinks. This data is called as hidden web. Based on calculations from a study done at University of California, Berkeley. It is estimated that the hidden web contains near about 91,850 tb and the surface web is only about 167 tb in 2003 [1]. Now recent studies estimated that 1.9 zetta bytes were reached and 0.3 zetta bytes were consumed worldwide in 2007 [2], An IDC report calculates that the total of all digital data created, replicated, and consumed will reach 6 zetta bytes in 2014 [3]. A significant portion of this large amount of data is estimated to be stored as structured or Relational data in web databases deep web makes up approximate $96 \%$ of all the content on the web, which is 500 to 550 times greater than the surface web [4]. It is an conformation to locate the deep web databases, because they are not registered with any type of search engines, are usually sparsely distributed, and always changing. To address this problem, previous work has proposed two types of crawlers, First one is generic crawlers and second is focused crawlers. Generic crawlers fetch all searchable forms and cannot focus on only specific topic.
Focused crawlers such as Form-Focused Crawler (FFC) focus on specific topic related to domain[5]. Adaptive Crawler for Hidden-web Entries (ACHE) can automatically search online databases on a specific topic. Crawler must produce a large quantity of high-quality results from the most relevant content sources [5]. For assessing quality source, Source ranks the results from the selected sources by computing the agreement between them [6]. When choosing a relevant subset from the available content sources, the set of retrieved forms is very heterogeneous. Thus it is necessary to develop enhanced crawling strategies that are able to quickly discover relevant content sources from the hidden web as much as possible. We propose an effective hidden web harvesting framework, namely Smart Crawler, for achieving both wide coverage and high efficiency for a Form focused crawler. Our crawler is divided into two stages 1)site locating and 2) in-site exploring.

\section{LITERATURE SURVEY}

\subsection{Crawling the hidden web}

Now days crawlers retrieve content only from the publically index able web so they ignore the tremendous amount of high quality content "hidden" behind search forms, In large searchable electronic databases. In this paper, here proposed crawler capable of extracting content from this hidden Web and also introduce a generic operational model of a hidden Web crawler and describe how this model is realized in hidden web expose[7]

\subsection{Deep web query extraction algorithm for information retrieval system}

Basically there are two types of web: surface web and deep web.

1)Surface web : easily accessible through conventional search engines so this information are static which means we get the same set of information with the same query.

2) Deep web: Not retrieved through conventional search engines. Deep web information is stored in searchable databases and these databases give results dynamically after processing user requests.

Because of this deep web there is large number of web databases available .That's why problem faced by users is data extraction corresponding to given query and also many results display for a submitted query. So for finding best deal user has to go through this long list which is very time consuming task. So in this paper system framework is prepare for solve problem of data extraction by ranking result.

For calculate ranking first calculated

1) Probabilities of attribute for query in particular user.

2) Probabilities of overall data.

3) Then mean of both probabilities is calculated.

In this way Ranking is done according to mean probability. 


\subsection{Hierarchical classification of web content}

The hierarchical structure is initially used to train different second-level classifiers for distinguish a second-level category from other categories within the same top level. Now a days very fast growth of information on the internet and intranets so it is becoming very difficult to find and organize relevant data. We can use machine learning techniques for text categorization, including multivariate regression models, Nearest neighbor classifiers, probabilistic Bayesian models, decision trees, neural networks, symbolic rule learning, and support vector achiness. These all approaches depend on having some initial labeled training data from this category models are learned. For Classification of web search result use classification technique which automatically organize search result into hierarchical structures by using training set of human labeled document and web categories, classification model can learn offline thus run time classification is vary and manual classification is easy to understand . For automatically classification technique two contains are important.[8]

1) Use short summary return from web search engine.

2) Focus on top level of the hierarchy.

Since we believe that many search results can be usefully disambiguated at this level.

\subsection{An interactive clustering based approach to integrating source query interfaces on the deep web}

Now a days increasing no of data sources are available on the web but their contents are only accessible through query interfaces. For integrating these sources, we consider the integration of their query interfaces. In this paper focus on the steps of integration of query interfaces[9].

1) General schema matching problem : Rather than matching two schemas at a time, here exploit the evidences from a large set of schemas at once for helping to identify mappings.

2) During the matching process, user interactions can be intro-duce, thus complementing the approaches where the user feedback is provided at the end of the matching process. 3) For identify complex mappings, it is possible to utilize both the structural and the instance-level .

4) Approach for the active learning of parameters constitutes an important step towards a systematic tuning of the parameters in schema matching algorithms.

\subsection{Optimal algorithm for crawling a hidden database in the web}

User access the data by issue queries through a search interface. Search engine can not effectively index hidden databases so access dada (which is store in hidden database from repository ) is unable to direct queries. In this paper, it remedies the problem by giving algorithms to extract all the tuples from a hidden database[10].

\subsection{Distributed search over the hidden web hierarchical database sampling and selection}

Many valuable text databases on the net have non-crawl able contents and they are hidden behind search interfaces. through unified query interface meta searchers are searching over many such database at once critical task for meta searchers to process query efficient and effectively is the selection of most promising databases for the query, a task that relies on summaries of the database content. In this paper here present an algorithm to derive content summaries by using 'focused query probes' from uncooperative database . Meta search engine is used for access the information in text database . It used to query multiple database simultaneously meta search engine perform three main task database selection, query translation, result merging . database selection of meta search engine is very crucial task in terms of query processing efficiently and effectiveness[11].

\subsection{An adaptive crawler for locating hidden web entry points}

In this paper describe new adaptive crawling strategies for hidden-Web sources to efficiently locate the entry points. The fact that locating hidden-Web is especially challenging because sources are very sparsely distributed. by using the contents of pages deals for problem to focus the crawl on a topic; by prioritizing promising links within the topic; and by also following links that may not lead to immediate benefit. In proposed a new framework crawlers automatically learn patterns of promising links and adapt their focus as the crawl progresses, thus reducing the amount of required manual setup and tuning. This shows that online learning pointing to significant gains in harvest rates-the adaptive crawlers retrieve up to three times as many forms as crawlers that use a fixed focus strategy[12]

\section{METHODOLOGY}

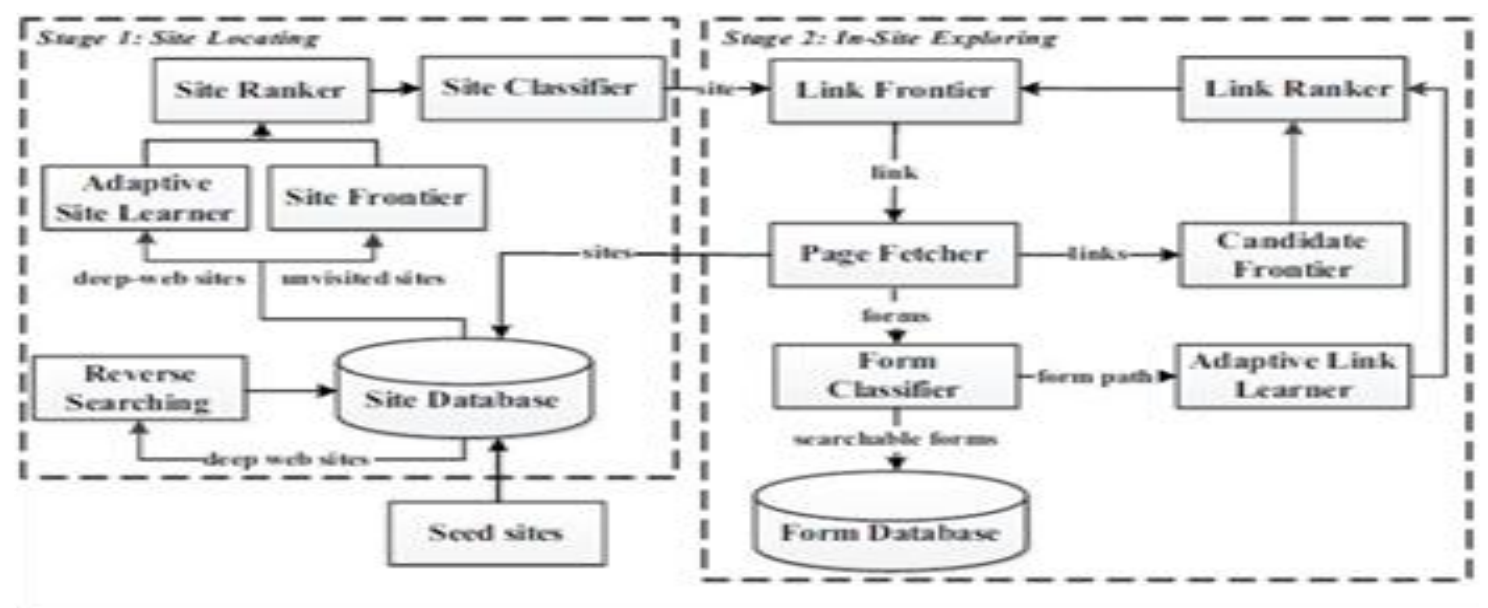

Fig 3.1 The Two stage architecture of Enhance Crawler 
In above fig stage 1 is site locating stage and stage 2 is in-site exploring stage

Now a day's wide web grows at a very large space so interest has been increased to locate web interfaces. Due to very high amount of web resources and dynamic nature of web achieving the wide coverage and high efficiency is become challenging issue. So for achieving this we proposed "Enhance crawler". This crawler architecture is divided into two part. In first part of crawler architecture i.e. site locating, The first site locating stage gives output the most relevant site for a given topic. the second part of crawler architecture is in-site exploring stage uncovers searchable forms from the site. Specifically, in the site locating stage search start with a seed set of sites i.e. candidate site in a site database. Candidate sites given for Crawler to start crawling, which begins by following URLs from chosen seed sites and explore other pages and other domains. When the number of unvisited URLs in the database is less than a threshold value during the crawling process, Enhanced Crawler performs "reverse searching" of known deep websites for getting center pages (highly ranked pages that have many links to the other domains) and feeds these pages back to the site database. Site Frontier is designed to fetch homepage of different URLs from the site database, and we are ranked and prioritize by Site Ranker on basis of relevant sites. The Site Ranker is improved by an Adaptive Site Learner, which adaptively learns from features of deep-web sites for accurate result. For getting more accurate results for a focused crawl, Classifier categorizes URLs into relevant or irrelevant for a given topic based on the homepage content. After finishing the work of a first stage i.e. relevant site searching, in the second stage work start on exploring and excavating searchable forms. In this case links of a most relevant sites are stored in link frontier and it's been used to fetch the corresponding pages. Additional links present in the link pages are been fed to candidate frontier, for prioritize and ranking links in candidate frontier and then Enhance crawler ranks them with the help of link ranker. Most Important point to notice here is site locating stage and in-site exploring stage are mutually intertwined. The Link Ranker is always adaptively improved by an Adaptive Link Learner, when a site crawling is completed feature of site is calculate by adaptive learner. Insite exploring contains two crawling strategies for high efficiency and wide coverage. Links within a site are prioritized with the help of Link Ranker and Form Classifier classifies all searchable forms and store in database.

\subsection{Algorithms used in proposed system}

***** Reverse searching for more sites. $* * * * *$

Input $=$ seed sites and harvested deep websites

Output $=$ relevant sites

while (of candidate sites less than a threshold) Do

\{

pick a deep website

site $=$ getDeepWebSite(siteDatabase, seedSites)

resultPage $=$ reverseSearch $($ site $)$

links $=$ extractLinks(resultPage)

foreach link in links

do \{

page $=$ downloadPage $($ link $)$

relevant $=$ classify $($ page $)$

if relevant

then

$\begin{array}{cc}\text { relevantSites } & \\ \text { extractUnvisitedSite(page) } & \text { Output relevantSites }\end{array}$

\subsection{Feature selection and ranking}

Enhance crawler encounters a variety of web pages during a crawling process and for efficiently crawling and wide coverage is depend upon ranking different sites and prioritizing links within a site.

\subsection{Adaptive learning}

Adaptive learning is educational method which uses computer as an interactive teaching devices and to orchestrate the allocation of human and mediated resources according to the unique need of every user. Enhance crawler uses an adaptive learning strategy that help to enhances the learning capacity during crawling. As shown in fig. no.1 site ranker and link ranker are periodically updated by adaptive learning.

\subsection{Ranking mechanism}

Enhance crawler uses a site URLs for prioritizing deep websites for a relevant topic, while doing ranking two main aspects are taken into consideration that are site similarity and site visit frequency. Site similarity calculate by checking the topic similarity between a new site and known deep web sites. Site frequency assessment parameter depends on the frequency site to appear in other website.

\section{CONCLUSION}

An effective gathering framework for deep-web interfaces, especially Enhance-Crawler is presented in this paper. In this paper, two levels of Enhance Crawler are presented: site finding and adjusted in-site investigating. Smart Crawler performs webpage based locating by conversely watching out 
the noted deep sites for focus pages, which might expeditiously gather several knowledge sources for thin domains. Enhance Crawler will achieves a great deal of right results by positioning collected destinations and focusing the crawl on a given topic. The in-webpage considering stage utilizes adaptive link ranking to take a look at interims of site and style a link tree for rejecting bias toward bound archives of site for more extensive range of web indexes. The usefulness of the projected two-phase crawler accomplishes higher harvest rates than another crawlers

\section{REFERENCES}

[1] Peter Lyman and Hal R. Varian. How much information? 2003. Technical report, UC Berkeley, 2003.

[2] Roger E. Bohn and James E. Short. How much information? 2009 report on American consumers. Technical report, University of California, San Diego, 2009.

[3] Idc worldwide predictions 2014: Battles for dominance and survival - on the 3rd platform. http://www.idc.com/ research/Predictions14/index.jsp, 2014.

[4] Michael K. Bergman. White paper: The deep web: Surfacing hidden value. Journal of electronic publishing, 7(1), 2001.

[5] Luciano Barbosa and Juliana Freire. Searching for hidden-web databases. In WebDB, pages 1-6, 2005.

[6] Balakrishnan Raju and Kambhampati Subbarao. Sourcerank: Relevance and trustassessment for deep web sourcesbased on inter-source agreement. In Proceedings of the 20th international conference on World Wide Web, pages 227-236, 2011.
[7] Sriram Raghavan and Hector Garcia-Molina. Crawling the hidden web. In Proceedings of the 27th International Conference on Very Large Data Bases, pages 129-138, 2000.

[8] Dumais Susan and Chen Hao. Hierarchical classification of Web content. In Proceedings of the 23rd Annual International ACM SIGIR conference on Research and Development in Information Retrieval, pages 256-263, Athens Greece, 2000

[9] Pages Wensheng Wu, Clement $\mathrm{Yu}$, AnHai Doan, and Weiyi Meng. An interactive clustering-based approach to integrating source query interfaces on the deep web. In Proceedings of the 2004 ACM SIGMOD international conference on Management of data, pages 95-106. ACM, 2004.

[10] Cheng Sheng, Nan Zhang, Yufei Tao, and Xin Jin. Optimal algorithms for crawling a hidden database in the web. Proceedings of the VLDB Endowment, 5(11):1112-1123, 2012.

[11] Panagiotis G Ipeirotis and Luis Gravano. Distributed search over the hidden web: Hierarchical database sampling and selection. In Proceedings of the 28th international conference on Very Large Data Bases, pages 394-405. VLDB Endowment, 2002.

[12] Mohamamdreza Khelghati, Djoerd Hiemstra, and Maurice Van Keulen. Deep web entity monitoring. In Proceedings of the 22nd international conference on World Wide Web companion, pages 377-382. International World Wide Web Conferences Steering Committee, 2013. 